\title{
Cervical spinal injury in children's community rugby football
}

\author{
G J Browne
}

Br J Sports Med 2006;40:68-71. doi: 10.1136/bjsm. 2005.020636

Correspondence to: GJ Browne, The Children's Hospital Institute of Sports Medicine, Westmead, Sydney, NSW, Australia; garyb@chw.edu.au

Accepted 12 July 2005

\begin{abstract}
Objective: To examine the characteristics of cervical spinal injury (CSI) in school aged children injured in community based rugby football who presented to the emergency department for assessment. Methods: This is a retrospective descriptive case series study reviewing the medical records of all children younger than 15 years of age who presented to the emergency department at the Children's Hospital at Westmead, Sydney, Australia for assessment of injury to the cervical spine between 2000 and 2003. Results: There were 125 children with CSI; most (97\%) were boys of mean age 12.7 years. Injuries occurred throughout the season, with an unexpected peak in June. Neck pain was the main presenting complaint (98\%). Neurological symptoms were reported in $43 \%$, half having concussion. Hyperextension of the neck accounted for a third of all cases and was usually the result of a spear tackle. Appropriate treatment of the cervical spine on the field of play before transport to hospital was inconsistently performed. Half of the players with CSI suffered secondary injuries, consisting of concussive head injury, faciomaxillary injury, eye injury, or limb fracture. Admission to hospital was common, with all children admitted undergoing further radiological assessment. Two minor fractures were reported and no permanent neurological disability. Overall, no adverse events were reported and the clinical outcome was good.

Conclusion: CSI in children playing rugby football is rarely catastrophic although often associated with other injuries. Continued efforts are needed to educate players and referees to prevent injury.
\end{abstract}

potential injury to the cervical spine between 2000 and 2003. This hospital is the designated paediatric spinal unit for the state of New South Wales in Australia. Included in the study were children involved in community based rugby football and who were identified on the field of play as having a potential injury to their cervical spine needing assessment at the specialised spinal unit. The study was approved by the hospital's ethics committee.

During the study period, there were 21248 trauma episodes, with 1745 episodes of CSI in children younger than 15 years. Of these, 1610 were due to motor vehicle and pedestrian accidents. There were 139 sports related CSIs, of which 125 were related to rugby. The remaining 14 were sustained in other activities such as trampoline, soccer, gymnastics, netball, baseball, and softball.

Injured children were assessed as to whether they needed urgent medical attention on the field of play by the coach/ teacher. As a standard requirement in Australia, all coaches and teachers in charge of physical education receive full training in first aid provided by the St Johns ambulance. They are also educated to assess the significance of an injury, particularly to the neck. Once there is any suspicion of significant injury or any potential injury to the neck, the NSW Ambulance Services is contacted to transport the injured child to a hospital for further assessment and treatment. While attending the injured child at the scene, the ambulance officer follows the standardised assessment and treatment protocol stipulated by the NSW Ambulance Services. At the same time, the emergency department of the receiving hospital is alerted that a patient with potential cervical spinal injury will be arriving. Once the patient arrives in the emergency department, treatment follows a predetermined clinical pathway.

In this study, comprehensive clinical information was collected using the emergency department clinical information system (Health-e-Care) in addition to the trauma information system. The patient characteristics collected included age, sex, nature and type of injury, and treatment 


\begin{tabular}{|c|c|}
\hline Characteristic & Number (\%) \\
\hline \multicolumn{2}{|l|}{ Age $^{*}$} \\
\hline $\begin{array}{l}\leqslant 10 \\
\geqslant 11\end{array}$ & $\begin{array}{l}27(22) \\
98(78)\end{array}$ \\
\hline \multicolumn{2}{|l|}{ Sex } \\
\hline M & $121(97)$ \\
\hline $\mathrm{F}$ & $4(3)$ \\
\hline \multicolumn{2}{|l|}{ Rugby rules } \\
\hline Union & 99 (79) \\
\hline League & $26(21)$ \\
\hline \multicolumn{2}{|l|}{ Mechanism of injury } \\
\hline Flexion & $37(30)$ \\
\hline Extension & $9(7)$ \\
\hline Flexion/extension & $16(13)$ \\
\hline Distraction & $7(6)$ \\
\hline Rotation & $3(2)$ \\
\hline Hyperextension & $44(35)$ \\
\hline Unknown & $9(7)$ \\
\hline \multicolumn{2}{|l|}{ Triage category } \\
\hline 1 & $5(4)$ \\
\hline 2 & $44(35)$ \\
\hline 3 & $52(42)$ \\
\hline 4 & $21(17)$ \\
\hline 5 & $3(2)$ \\
\hline \multicolumn{2}{|l|}{ Neck pain } \\
\hline Yes & $115(98)$ \\
\hline No & $2(2)$ \\
\hline \multicolumn{2}{|l|}{ Concussion } \\
\hline Yes & $29(23)$ \\
\hline No & 95 (77) \\
\hline \multicolumn{2}{|l|}{ Neurological symptoms } \\
\hline Yes & $53(43)$ \\
\hline No & $69(57)$ \\
\hline \multicolumn{2}{|l|}{ Other injuries } \\
\hline Yes & $68(54)$ \\
\hline No & $57(46)$ \\
\hline \multicolumn{2}{|l|}{ Treatment at scene } \\
\hline Yes & $96(82)$ \\
\hline No & $21(18)$ \\
\hline \multicolumn{2}{|l|}{ Clinical evaluation at ED } \\
\hline Assessment \& observation only & $20(16)$ \\
\hline Imaging & $105(84)$ \\
\hline \multicolumn{2}{|l|}{ Surgery required } \\
\hline Yes & 0 \\
\hline No & $125(100)$ \\
\hline \multicolumn{2}{|l|}{ Admission } \\
\hline Yest & $39(31)$ \\
\hline No & $86(69)$ \\
\hline
\end{tabular}

on the field and in the emergency department. The following clinical outcomes were reported: admission to hospital, length of stay in the emergency department, morbidity (spinal cord or bony injury), co-morbidity (injuries other than CSI), and mortality from CSI.

Data were analysed descriptively using frequencies and percentages. As all variables of interest in this study were categorical variables, the Pearson $\chi^{2}$ test for the association between patients' characteristics and admission was used. If the expected values in any one of the cells were less than 5, Fisher's exact test was used.

\section{RESULTS}

There were 125 children who injured their cervical spine while playing rugby football during the study period. Of these 125 cases, 99 were the result of playing rugby league and 26 were from playing rugby union. Table 1 summarises the results. Most $(n=121,97 \%)$ rugby football CSIs occurred in boys who were over 11 years of age $(\mathrm{n}=98,78 \%)$ (mean (SD) age 12.7 (2.1) years). Injury incidence was consistent throughout the season, although a third were reported as occurring in June $(\mathrm{p}<0.001)$ (fig 1$)$. There were no trends in player position and injury, as permanent positions were rarely allocated, although players tended to be in either the forward pack or back row.

Neck pain was reported in $98 \%$ of presentations and was the main complaint of CSI reported at the scene or on the field of play by attending ambulance officers. Associated neurological symptoms were reported in 53 cases (43\%), and concussion was reported in $39(23 \%)$. Of those patients suffering a concussion, $27(69 \%)$ had a brief alteration in mental state or confusion, and $12(31 \%)$ were reported to have an associated loss of consciousness. Transient sensory, motor, or general paralysis was also reported in 14 cases; 10 had transient sensory or motor deficit, and four had transient quadriparesis.

Further review of the ambulance reports indicated that treatment at the scene, which consisted of immobilisation of the cervical spine, occurred in 96 cases $(82 \%)$.

Resuscitation on the field, consisting of oxygen and fluid administration, was reported in one rugby league player who had a seizure on the field of play.

The mechanisms of injury were extension of the neck (53; $37 \%)$, flexion of the neck $(37 ; 30 \%)$, flexion/extension ( 16 ; $13 \%$ ), distraction of the neck (seven; $6 \%$ ), and a rotation type injury (three; $2 \%)$. In nine $(7 \%)$ cases, the mechanism of injury was unreported. Further examination of the nature of injury indicated that, of the 46 cases of extension injury, $27 \%$ were hyperextension injuries that occurred as a result of a spear tackle. This type of injury was reported exclusively in players of rugby league. Of the 37 cases of flexion injury to the neck, $23 \%$ occurred during a scrum while playing rugby union. Thirty five of these players reported that they were either filling in for an absent player or trying out a new position at the time of injury.

There was a significant association between rugby codes and the nature of injury. A rugby union player was more likely to have sustained a flexion/extension injury (81\%) than a rugby league player $\left(41 \% ; \chi_{1}^{2}=12.76, p<0.001\right)$. Alternatively rotation/hyperextension injury was more commonly reported in rugby league players with a hyperextension injury, many the result of a spear tackle. A significant association was also found between rugby codes and the incidence of concussion: $27 \%$ of rugby league players $v 8 \%$ of rugby union players $\left(\chi_{1}^{2}=4.14, p=0.042\right)$. No other variables were found to be associated with rugby rules. Because of the lack of difference in all of the other variables, the two rugby codes were combined for the analysis of outcomes.

The occurrence of co-morbidity in players of rugby football was significant. A concussive head injury was reported to occur in $29(23 \%)$ of all rugby players with a CSI. Additional co-morbid injuries with a CSI included fractures to the maxilla, orbit, arm, or leg in six (5\%), laceration to the face/ scalp in $24(19 \%)$, epistaxis in six (5\%), and hyphaema in two $(2 \%)$ cases.

Of the 125 children with rugby football related CSI, 20 $(16 \%)$ were observed in the emergency department without any further investigation. The remaining 105 (84\%) required imaging, which include cervical spine radiology in all, computed tomography scan in $22(18 \%)$, and magnetic resonance imaging in $10(8 \%)$. Although $39(31 \%)$ patients were admitted to hospital, none required surgical intervention.

Of the 39 patients admitted to hospital, admission was for CSI injury alone in 17 (14\%). In 22 (18\%) patients, admission was for treatment of co-morbid conditions, with laceration repair accounting for $15(68 \%)$. The mean length of stay for all admitted patients was 43.4 hours with a median of 48 hours. Morbidity was low, with two cases of isolated 


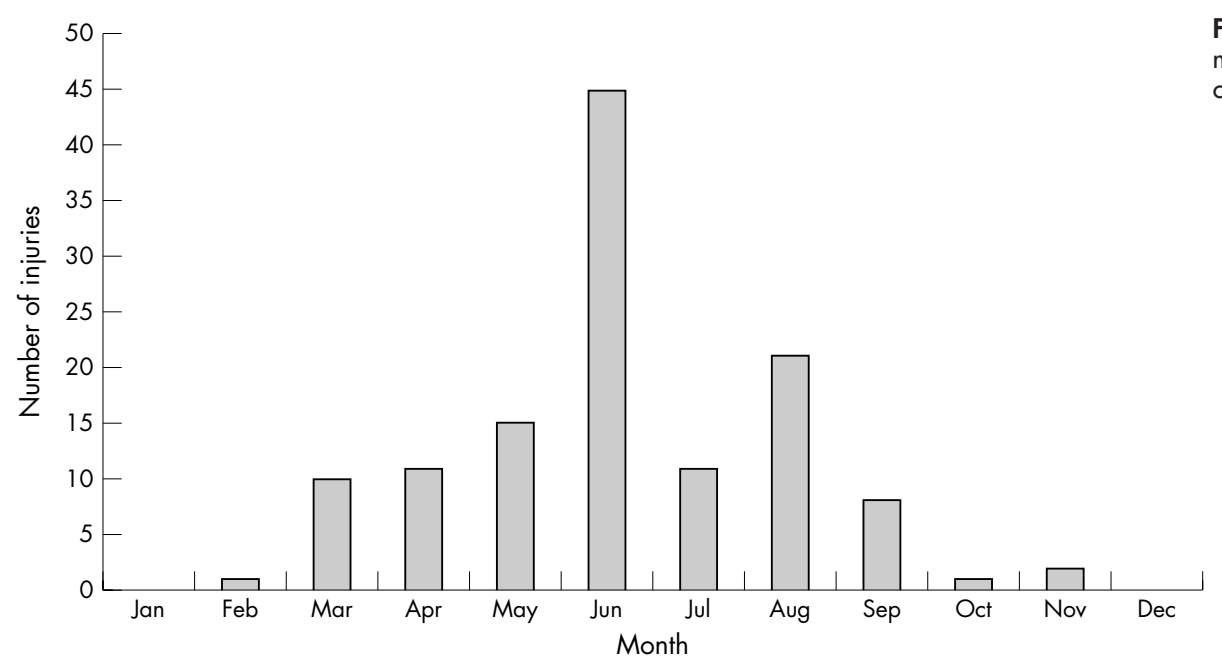

Figure 1 Time of presentation by month of the year for all rugby football cervical spine injuries in school boys.

fracture to the spinous process (C5 and C6 respectively) treated in hospital conservatively. Both players were from the rugby league code and suffering a hyperextension injury to the neck (spear tackle). No deaths were reported.

Some patient characteristics were identified to be associated with admission to hospital (table 2). These included neurological symptoms, other injuries, treatment at the scene, and imaging in the emergency department.

\section{DISCUSSION}

Many studies have reported on acute CSI in football, but this is the first to report on acute CSI in non-elite (community based) rugby football players of school age. Most of these injuries were found to be potential CSI in which a strain or sprain occurs without damage to the spinal cord or surrounding vertebral column. The most common mechanism of injury was hyperextension. It resulted in two stable fractures to the lower cervical spine. These were uncomplicated fractures which responded well to conservative treatment. No long term adverse events were reported in relation to the primary CSI. A quarter of cases required admission to hospital for treatment of secondary injury, which included concussion, maxillofacial injury, eye injury, and limb fracture.

In this study, rugby union was more likely to be associated with a flexion/extension mechanism of injury. This is consistent with other studies which found that rugby union players were more likely to suffer CSI in a collapsed scrum. ${ }^{2}{ }^{3}$ Conversely rugby league was found to have a significant association with rotation/hyperextension injury, which in most cases resulted from a spear tackle. However, no significant differences in clinical outcomes were found between the two rugby codes. A possible explanation is that young immature teenagers are unlikely to cause a collision severe enough to result in serious injury to the spine. This, however, remains to be confirmed. The spear tackle was a common cause of injury in the rugby league players. This

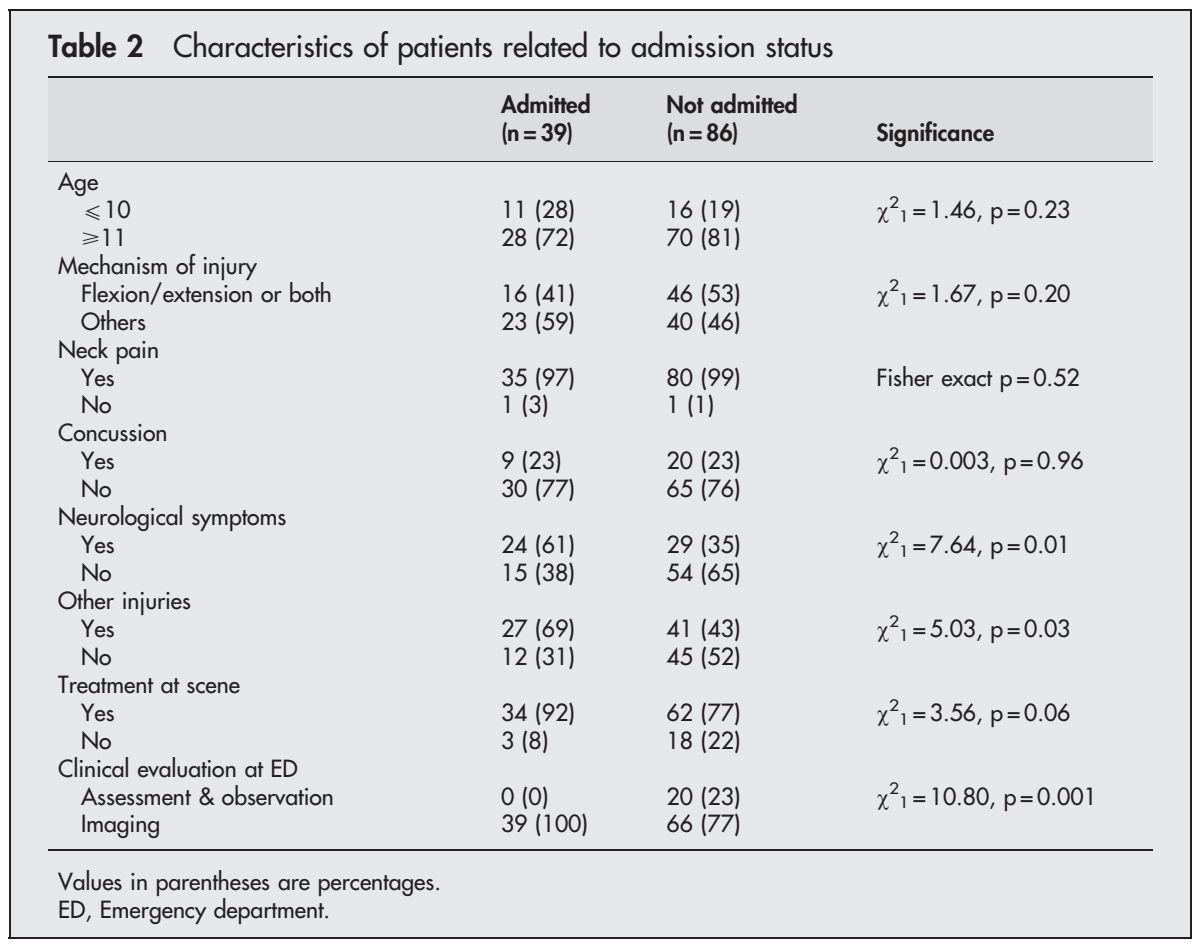


What is already known on this topic

- Cervical spine injury in children is relatively rare

- In elite rugby, interventions to improve player safety have possibly reduced the incidence of cervical spine injury but no data are available for school aged rugby football players

suggests that better adherence to the rules of rugby league may prevent most of these injuries.

Studies on adult players have reported an increase in frequency of CSI early in the season, ${ }^{7}$ but this trend was not shown in the present study. CSIs were spread throughout the season except for an unexpected peak in June, which is probably due to heavy exposure to rugby in that month.

Although the position of the player when injured is not well reported in this study, most CSIs occurred in the scrum for rugby union and during a tackle in rugby league. Tackle injuries in league were of particular concern as many were the result of the illegal spear tackle. ${ }^{9}{ }^{10}$ Here education and leadership from elite players may be important in preventing these injuries. ${ }^{11}$

Precautions must be taken in every case of a potential CSI. In this study, treatment on the field of play was not effectively applied in over a quarter of cases, with no spinal immobilisation before transport to hospital in these players. Recent reports highlight the need to review policies and practices in New South Wales in relation to sporting injuries. ${ }^{12}$ One study in particular reported a lack of policies for the effective treatment of head and neck injuries on the field of play in children. ${ }^{13}$ The results from the present study support the need for policies for on-field injury management to be put in place so that more effective treatment is delivered to players with potential CSI before they are transported to hospital for assessment.

Spinal cord injuries are at one end of a spectrum of injury in football. An association of CSI with secondary injury is well reported in the literature, particularly concussive head injury. ${ }^{78}$ In this study, over half of the players had suffered an injury in addition to the primary CSI. These associated injuries were concussive head injury, limb fractures, or facial, maxillary, and/or eye injuries. A quarter of children with secondary injury were admitted to hospital for further treatment of that injury. Associated secondary injury adds to the considerable overall burden of CSI (even if the spine is cleared of trauma) to the healthcare system.

Given the current nature of the paediatric trauma system in Sydney, most suspected cases of CSI in children are eventually assessed in hospital. This has provided a convenient sample which includes nearly all paediatric CSI patients in the western region of Sydney, where most of the city's children reside. One limitation of this study is report bias, as in most cases the mechanism of injury was obtained from the patient or parent. In the few cases where this information could not be obtained directly, it was obtained by
What this study adds

- Cervical spine injury in children playing rugby football is rarely catastrophic although often associated with other injuries

- Continued efforts are needed to educate players and referees to prevent injury

proxy. This may result in some report bias in the data collection. A second limitation of this study is that only players who presented to the emergency department were included. As these are the most seriously injured players, care must be taken in extrapolating these results to the general rugby playing population. This may have introduced bias, which could subsequently affect recommendations for any injury prevention.

CSI in children playing rugby football is rarely catastrophic. Some of these injuries are uncomplicated fractures which respond well to conservative treatment. In this study, secondary injury accounted for the greatest morbidity. Continued efforts should focus on education and more effective use of safety equipment.

\section{ACKNOWLEDGEMENTS}

This project was supported by The Children's Hospital at Westmead and all data were collected in the emergency department. I acknowledge my emergency and trauma colleagues who gave their support during the collection of these data.

Competing interests: none declared

\section{REFERENCES}

1 Armour KS, Clatworthy BJ, Bean AR, et al. Spinal injuries in New Zealand rugby and rugby league: a twenty-year survey. N Z Med J 1997; 1 10:462-5.

2 Burry HC, Calcinai CJ. The need to make rugby safer. Br Med J (Clin Res Ed) 1988;296:149-50.

3 Taylor TKF, Coolican M, Parker RJ, et al. Spinal cord injuries in Australian footballers. J Bone Joint Surg [Br] 2004;86(suppl IV):489.

4 Rotem TR, Lawson JS, Wilson SW, et al. Severe cervical spinal cord injuries related to rugby union and league football in New South Wales, 1984-96. Med J Aust 1998; 168:379-81.

5 Quarrie KL, Cantu RC, Chalmers DJ. Rugby union injuries to the cervical spine and spinal cord. Sports Med 2002;32:633-53.

6 Scher AT. Rugby injuries to the cervical spine and spinal cord: a 10-year review. Clin Sports Med 1998;17:195-206.

7 Tayler TKF. Spinecare Foundation and the Australian Spinal Cord injury Units. Spinal cord injuries in Australian footballers. ANZ J Surg 2003;73:493-9.

8 Taylor TKF, Coolican MRJ. Spinal-cord injuries in Australian footballers, 1960-85. Med J Aust 1987;147:112-18.

9 Brigham CD, Warren R. Head to head on spear tackler's spine: criteria and implications for return to play. J Bone Joint Surg [Am] 2003;85:381-2.

10 Cantu RC, Mueller FO. Catastrophic football injuries: 1977-1998. Neurosurgery 2000;47:673-7.

11 Noakes TD, Jakoet I, Baalbergen E. An apparent reduction in the incidence and severity of spinal cord injuries in schoolboy rugby players in the Western Cape since 1990. South Afr Med J 1999;89:540-5.

12 Davis PM, McKelvey MK. Medicolegal aspects of athletic cervical spine injury. Clin Sports Med 1998;17:147-54.

13 Donaldson A, Forero R, Finch C. The first aid policies and practices of community sports clubs in northern Sydney, Australia. Health Promot J Austr 2004; 15:155-61. 